\title{
Eigenvalues of quasibounded maximal monotone operators
}

In-Sook Kim ${ }^{*}$ and Jung-Hyun Bae

\author{
${ }^{*}$ Correspondence: iskim@skku.edu \\ Department of Mathematics, \\ Sungkyunkwan University, Suwon, \\ 440-746, Republic of Korea
}

\begin{abstract}
Let $X$ be a real reflexive separable Banach space with dual space $X^{*}$ and let $L$ be a dense subspace of $X$. We study a nonlinear eigenvalue problem of the type

$$
0 \in T x+\lambda C x
$$

where $T: D(T) \subset X \rightarrow 2^{X^{*}}$ is a strongly quasibounded maximal monotone operator and $C: D(C) \subset X \rightarrow X^{*}$ satisfies the condition $\left(S_{+}\right)_{D(C)}$ with $L \subset D(C)$. The method of approach is to use a topological degree theory for $\left(S_{+}\right)_{L}$-perturbations of strongly quasibounded maximal monotone operators, recently developed by Kartsatos and Quarcoo. Moreover, applying degree theory, a variant of the Fredholm alternative on the surjectivity of the operator $\lambda T+C$ is discussed, where we assume that $\lambda$ is not an eigenvalue for the pair $(T, C), T$ and $C$ are positively homogeneous, and $C$ satisfies the condition $\left(S_{+}\right)_{L}$
\end{abstract}

\section{Introduction and preliminaries}

A systematic theory of compact operators emerged from the theory of integral equations of the form

$$
T x+\lambda x=y, \quad \text { where } T x(t)=\int_{a}^{b} k(t, s, x(s)) d s .
$$

Here, $\lambda \in \mathbb{R}$ is a parameter, $y$ and $k$ are given functions, and $x$ is the unknown function. Such equations play a role in the theory of differential equations. The study goes back to Krasnosel'skii [1]. Moreover, the eigenvalue problem of the form

$$
T x+\lambda C x=0
$$

could be solved with the Galerkin method, where $C$ is continuous, bounded, and of type (S); see, e.g., [2].

From now on, we concentrate on the class of maximal monotone operators, as a generalization of linear self-adjoint operators. The theory of nonlinear maximal monotone operators started with a pioneer work of Minty [3] and has been extensively developed, with applications to evolution equations and to variational inequalities of elliptic and parabolic type; see $[4,5]$. The eigenvalue problem for various types of nonlinear operators was investigated in [6-10]. As a key tool, topological degree theory was made frequent use of; for instance, the Leray-Schauder degree and the Kartsatos-Skrypnik degree; see [11-15].

\section{Springer}

(02014 Kim and Bae; licensee Springer. This is an Open Access article distributed under the terms of the Creative Commons Attribution License (http://creativecommons.org/licenses/by/2.0), which permits unrestricted use, distribution, and reproduction in any medium, provided the original work is properly cited. 
Let $X$ be a real reflexive Banach space with dual space $X^{*}$. We consider a nonlinear eigenvalue problem of the form

$0 \in T x+\lambda C x$

where $T: D(T) \subset X \rightarrow 2^{X^{*}}$ is a maximal monotone multi-valued operator and $C: D(C) \subset$ $X \rightarrow X^{*}$ is a single-valued operator. In the case where the operator $C$ or the resolvents of $T$ are compact, it was studied in $[6,7,10]$ by using the Leray-Schauder degree for compact operators. When the operator $C$ is densely defined and quasibounded and satisfies the condition $\left(\tilde{S}_{+}\right)$, Kartsatos and Skrypnik [9] solved the above problem (E) via the topological degree for these operators given in [13].

We are now focused on the quasiboundedness of the operator $T$ instead of that of the operator $C$. Actually, a strongly quasibounded operator due to Browder and Hess [16] may not necessarily be bounded. One more thing to be considered is the condition $\left(S_{+}\right)_{L}$, where $L$ is a dense subspace of $X$ with $L \subset D(C)$. In fact, the condition $\left(S_{+}\right)_{0, L}$ was first introduced in [12] and the structure of the class $\left(S_{+}\right)_{L}$ or $\left(S_{+}\right)_{D(C)}$ was discussed in [17], as a natural extension of the class $\left(S_{+}\right)$; see $[4,14]$.

In the present paper, the first goal is to study the above eigenvalue problem (E) for strongly quasibounded maximal monotone operators, provided that the operator $C$ satisfies the condition $\left(S_{+}\right)_{D(C)}$. In addition, we assume the following property $(\mathcal{P})$ : For $\epsilon>0$, there exists a $\lambda>0$ such that the inclusion

$$
0 \in T x+\lambda C x+\varepsilon J x
$$

has no solution in $D(T) \cap D(C) \cap \Omega$, where $\Omega$ is a bounded open set in $X$ and $J$ is a normalized duality operator. This property is closely related to the use of a topological tool for finding the eigensolution on the boundary of $\Omega$; see $[9,10]$. To solve the above problem (E), we thus use the degree theory for densely defined $\left(S_{+}\right)_{L}$-perturbations of maximal monotone operators introduced by Kartsatos and Quarcoo in [18]. Roughly speaking, the degree function is based on the Kartsatos-Skrypnik degree [8] of the densely defined operators $T_{t}+C$, which is constant for all small values of $t$, where $T_{t}$ is the approximant introduced by Brézis et al. [19]. Such an approach was first used by Browder in [11]. The second goal is to establish a variant of a Fredholm alternative result on the surjectivity for the operator $\lambda T+C$, where $\lambda \geq 1$ is not an eigenvalue for the pair $(T, C)$ and the operator $C$ satisfies the condition $\left(S_{+}\right)_{L}$; see $[9,20]$.

This paper is organized as follows: In Section 2, we give some eigenvalue results for strongly quasibounded maximal monotone operators by applying the Kartsatos-Quarcoo degree theory. Section 3 contains a version of the Fredholm alternative for positively homogeneous operators, with a regularization method by means of a duality operator $J_{\varphi}$.

Let $X$ be a real Banach space, $X^{*}$ its dual space with the usual dual pairing $\langle\cdot, \cdot\rangle$, and $\Omega$ a nonempty subset of $X$. Let $\bar{\Omega}$, int $\Omega$, and $\partial \Omega$ denote the closure, the interior, and the boundary of $\Omega$ in $X$, respectively. The symbol $\rightarrow(\rightarrow)$ stands for strong (weak) convergence. An operator $A: \Omega \rightarrow X^{*}$ is said to be bounded if $A$ maps bounded subsets of $\Omega$ into bounded subsets of $X^{*}$. A is said to be demicontinuous if, for every $x_{0} \in \Omega$ and for every sequence $\left\{x_{n}\right\}$ in $\Omega$ with $x_{n} \rightarrow x_{0}$, we have $A x_{n} \rightarrow A x_{0}$. 
An operator $T: D(T) \subset X \rightarrow 2^{X^{*}}$ is said to be monotone if

$$
\left\langle u^{*}-v^{*}, x-y\right\rangle \geq 0 \quad \text { for every } x, y \in D(T) \text { and every } u^{*} \in T x, v^{*} \in T y \text {, }
$$

where $D(T)=\{x \in X: T x \neq \emptyset\}$ denotes the effective domain of $T$.

The operator $T$ is said to be maximal monotone if it is monotone and it follows from $\left(x, u^{*}\right) \in X \times X^{*}$ and

$$
\left\langle u^{*}-v^{*}, x-y\right\rangle \geq 0 \quad \text { for every } y \in D(T) \text { and every } v^{*} \in T y
$$

that $x \in D(T)$ and $u^{*} \in T x$.

An operator $T: D(T) \subset X \rightarrow 2^{X^{*}}$ is said to be strongly quasibounded if for every $S>0$ there exists a constant $K(S)>0$ such that for all $x \in D(T)$ with

$$
\|x\| \leq S \quad \text { and } \quad\left\langle u^{*}, x\right\rangle \leq S
$$

where $u^{*} \in T x$, we have $\left\|u^{*}\right\| \leq K(S)$.

We say that $T: D(T) \subset X \rightarrow 2^{X^{*}}$ satisfies the condition $\left(S_{q}\right)$ on a set $M \subset D(T)$ if for every sequence $\left\{x_{n}\right\}$ in $M$ with $x_{n} \rightarrow x_{0}$ and every sequence $\left\{u_{n}^{*}\right\}$ with $u_{n}^{*} \rightarrow u^{*}$ where $u_{n}^{*} \in T x_{n}$, we have $x_{n} \rightarrow x_{0}$.

We say that $T: D(T) \subset X \rightarrow X^{*}$ satisfies the condition $\left(S_{+}\right)$on a set $M \subset D(T)$ if for every sequence $\left\{x_{n}\right\}$ in $M$ with

$$
x_{n} \rightarrow x_{0} \quad \text { and } \quad \limsup _{n \rightarrow \infty}\left\langle T x_{n}, x_{n}-x_{0}\right\rangle \leq 0,
$$

we have $x_{n} \rightarrow x_{0}$.

Throughout this paper, $X$ will always be an infinite-dimensional real reflexive separable Banach space which has been renormed so that $X$ and its dual $X^{*}$ are locally uniformly convex.

An operator $J_{\varphi}: X \rightarrow X^{*}$ is said to be a duality operator if

$$
\left\langle J_{\varphi} x, x\right\rangle=\varphi(\|x\|)\|x\| \quad \text { and } \quad\left\|J_{\varphi} x\right\|=\varphi(\|x\|) \quad \text { for } x \in X,
$$

where $\varphi:[0, \infty) \rightarrow[0, \infty)$ is continuous, strictly increasing, $\varphi(0)=0$ and $\varphi(t) \rightarrow \infty$ as $t \rightarrow \infty$. When $\varphi$ is the identity map $I, J:=J_{I}$ is called a normalized duality operator.

It is described in [21] that $J_{\varphi}$ is continuous, bounded, surjective, strictly monotone, maximal monotone, and that it satisfies the condition $\left(S_{+}\right)$on $X$.

The following properties as regards maximal monotone operators will often be used, taken from [19, Lemma 1.3], [13, Lemma 3.1], [22, Lemma 1], and [18, Lemma D] in this order.

Lemma 1.1 Let $T: D(T) \subset X \rightarrow 2^{X^{*}}$ be a maximal monotone operator. Then the following statements hold:

(a) For each $t \in(0, \infty)$, the operator $T_{t} \equiv\left(T^{-1}+t J^{-1}\right)^{-1}: X \rightarrow X^{*}$ is bounded, demicontinuous, and maximal monotone.

(b) If, in addition, $0 \in D(T)$ and $0 \in T(0)$, then the operator $(0, \infty) \times X \rightarrow X^{*}$, $(t, x) \mapsto T_{t} x$ is continuous on $(0, \infty) \times X$. 
Lemma 1.2 Let $T: D(T) \subset X \rightarrow 2^{X^{*}}$ and $S: D(S) \subset X \rightarrow X^{*}$ be two maximal monotone operators with $0 \in D(T) \cap D(S)$ and $0 \in T(0) \cap S(0)$ such that $T+S$ is maximal monotone. Assume that there is a sequence $\left\{t_{n}\right\}$ in $(0, \infty)$ with $t_{n} \downarrow 0$ and a sequence $\left\{x_{n}\right\}$ in $D(S)$ such that $x_{n} \rightarrow x_{0} \in X$ and $T_{t_{n}} x_{n}+w_{n}^{*} \rightarrow y_{0}^{*} \in X^{*}$, where $w_{n}^{*} \in S x_{n}$. Then the following hold:

(a) The inequality $\liminf _{n \rightarrow \infty}\left\langle T_{t_{n}} x_{n}+w_{n}^{*}, x_{n}-x_{0}\right\rangle \geq 0$ is true.

(b) If $\lim _{n \rightarrow \infty}\left\langle T_{t_{n}} x_{n}+w_{n}^{*}, x_{n}-x_{0}\right\rangle=0$, then $x_{0} \in D(T+S)$ and $y_{0}^{*} \in(T+S) x_{0}$.

Lemma 1.3 Let $T: D(T) \subset X \rightarrow 2^{X^{*}}$ be a strongly quasibounded maximal monotone operator such that $0 \in D(T)$ and $0 \in T(0)$. If $\left\{t_{n}\right\}$ is a sequence in $(0, \infty)$ and $\left\{x_{n}\right\}$ is a sequence in $X$ such that

$$
\left\|x_{n}\right\| \leq S \text { and }\left\langle T_{t_{n}} x_{n}, x_{n}\right\rangle \leq S_{1}
$$

where $S, S_{1}$ are positive constants, then the sequence $\left\{T_{t_{n}} x_{n}\right\}$ is bounded in $X^{*}$.

Let $L$ be a dense subspace of $X$ and let $\mathcal{F}(L)$ denote the class of all finite-dimensional subspaces of $L$. Let $\left\{F_{n}\right\}$ be a sequence in the class $\mathcal{F}(L)$ such that for each $n \in \mathbb{N}$

$$
F_{n} \subset F_{n+1}, \quad \operatorname{dim} F_{n}=n, \quad \text { and } \overline{\bigcup_{n \in \mathbb{N}} F_{n}}=X .
$$

Set $L\left\{F_{n}\right\}:=\bigcup_{n \in \mathbb{N}} F_{n}$.

Definition 1.4 Let $C: D(C) \subset X \rightarrow X^{*}$ be a single-valued operator with $L \subset D(C)$. We say that $C$ satisfies the condition $\left(S_{+}\right)_{0, L}$ if for every sequence $\left\{F_{n}\right\}$ in $\mathcal{F}(L)$ satisfying equation (1.1) and for every sequence $\left\{x_{n}\right\}$ in $L$ with

$$
x_{n} \rightarrow x_{0}, \quad \limsup _{n \rightarrow \infty}\left\langle C x_{n}, x_{n}\right\rangle \leq 0, \quad \text { and } \quad \lim _{n \rightarrow \infty}\left\langle C x_{n}, y\right\rangle=0
$$

for every $y \in L\left\{F_{n}\right\}$, we have $x_{n} \rightarrow x_{0}, x_{0} \in D(C)$, and $C x_{0}=0$.

We say that $C$ satisfies the condition $\left(S_{+}\right)_{L}$ if the operator $C_{h}: D(C) \rightarrow X^{*}$, defined by $C_{h} x:=C x-h$, satisfies the condition $\left(S_{+}\right)_{0, L}$ for every $h \in X^{*}$.

We say that the operator $C$ satisfies the condition $\left(S_{+}\right)_{0, D(C)}$ if it satisfies the condition $\left(S_{+}\right)_{0, L}$ with ' $\left\{x_{n}\right\} \subset L$ ' replaced by ' $\left\{x_{n}\right\} \subset D(C)$ '. We say that $C$ satisfies the condition $\left(S_{+}\right)_{D(C)}$ if the operator $C_{h}$ satisfies the condition $\left(S_{+}\right)_{0, D(C)}$ for every $h \in X^{*}$.

It is obvious from Definition 1.4 that if the operator $C$ satisfies the condition $\left(S_{+}\right)_{D(C)}$, then $C$ satisfies the condition $\left(S_{+}\right)_{L}$. However, the converse is not true in general, as we see in Example 3.2 of [17].

\section{The existence of eigenvalues}

In this section, we deal with some eigenvalue results for strongly quasibounded maximal monotone operators in reflexive separable Banach spaces, based on a topological degree theory for $\left(S_{+}\right)_{L}$-perturbations of maximal monotone operators due to Kartsatos and Quarcoo [18].

We establish the existence of an eigenvalue concerning $\left(S_{+}\right)_{D(C)}$-perturbations of strongly quasibounded maximal monotone operators. 
Theorem 2.1 Let $\Omega$ be a bounded open set in $X$ with $0 \in \Omega$ and let $L$ be a dense subspace of $X$. Suppose that $T: D(T) \subset X \rightarrow 2^{X^{*}}$ is a multi-valued operator and $C: D(C) \subset X \rightarrow X^{*}$ is a single-valued operator with $L \subset D(C)$ such that

(t1) $T$ is maximal monotone and strongly quasibounded with $0 \in D(T)$ and $0 \in T(0)$,

(c1) $C$ satisfies the condition $\left(S_{+}\right)_{D(C)}$,

(c2) for every $F \in \mathcal{F}(L)$ and $v \in L$, the function $c(F, v): F \rightarrow \mathbb{R}$, defined by $c(F, v)(x)=\langle C x, v\rangle$, is continuous on $F$, and

(c3) there exists a nondecreasing function $\psi:[0, \infty) \rightarrow[0, \infty)$ such that

$$
\langle C x, x\rangle \geq-\psi(\|x\|) \text { for all } x \in D(C)
$$

Let $\Lambda$ and $\varepsilon_{0}$ be two given positive numbers.

(a) For a given $\varepsilon>0$, assume the following property $(\mathcal{P})$ :

There exists a $\lambda \in(0, \Lambda]$ such that the inclusion

$$
0 \in T x+\lambda C x+\varepsilon J x
$$

has no solution in $D(T+C) \cap \Omega$.

Then there exists a $\left(\lambda_{\varepsilon}, x_{\varepsilon}\right) \in(0, \Lambda] \times(D(T+C) \cap \partial \Omega)$ such that

$$
0 \in T x_{\varepsilon}+\lambda_{\varepsilon} C x_{\varepsilon}+\varepsilon J x_{\varepsilon} .
$$

Here, $D(T+C)$ denotes the intersection of $D(T)$ and $D(C)$.

(b) If property $(\mathcal{P})$ is fulfilled for every $\varepsilon \in\left(0, \varepsilon_{0}\right]$, $T$ satisfies the condition $\left(S_{q}\right)$ on $D(T) \cap \partial \Omega, 0 \notin T(D(T) \cap \partial \Omega)$, and the set $C(D(C) \cap \partial \Omega)$ is bounded, then the inclusion

$$
0 \in T x+\lambda C x
$$

has a solution $\left(\lambda_{0}, x_{0}\right)$ in $(0, \Lambda] \times(D(T+C) \cap \partial \Omega)$.

Proof (a) Assume that the conclusion of (a) is not true. Then for every $\lambda \in(0, \Lambda]$, the following boundary condition holds:

$$
0 \notin T x+\lambda C x+\varepsilon J x \quad \text { for all } x \in D(T+C) \cap \partial \Omega .
$$

Considering a multi-valued map $H$ given by

$$
H(s, x):=T x+s \Lambda C x+\varepsilon J x \text { for } s \in[0,1],
$$

the inclusion $0 \in H(s, x)$ has no solution $x$ in $D(T+C) \cap \partial \Omega$ for all $s \in[0,1]$. Actually, this holds for $s=0$, in view of the injectivity of the operator $T+\varepsilon J$ with $0 \in(T+\varepsilon J)(D(T) \cap \Omega)$.

Now we consider a single-valued map $H_{1}$ given by

$$
H_{1}(t, s, x):=T_{t} x+s \Lambda C x+\varepsilon J x \quad \text { for } t \in(0, \infty) \text { and } s \in[0,1] .
$$


We will first show that there exists a positive number $t_{0}$ such that the equation

$$
H_{1}(t, s, x)=0
$$

has no solution $x$ in $D(C) \cap \partial \Omega$ for all $t \in\left(0, t_{0}\right]$ and all $s \in[0,1]$. For $s=0$, assertion (2.2) is obvious because $\left(T_{t}+\varepsilon J\right) x=0$ implies $x=0$. Assume that assertion (2.2) does not hold for any $s \in(0,1]$. Then there exist sequences $\left\{t_{n}\right\}$ in $(0, \infty),\left\{s_{n}\right\}$ in $(0,1]$, and $\left\{x_{n}\right\}$ in $D(C) \cap \partial \Omega$ such that $t_{n} \downarrow 0, s_{n} \rightarrow s_{0}, x_{n} \rightarrow x_{0}, J x_{n} \rightarrow j^{*}$, and

$$
T_{t_{n}} x_{n}+s_{n} \Lambda C x_{n}+\varepsilon J x_{n}=0,
$$

where $s_{0} \in[0,1], x_{0} \in X$, and $j^{*} \in X^{*}$. Let $S$ be a positive upper bound for the bounded sequence $\left\{\left\|x_{n}\right\|\right\}$. Note that $s_{0} \in(0,1]$. Indeed, if $s_{0}=0$, then we have by the monotonicity of $T_{t_{n}}$ with $T_{t_{n}}(0)=0$, equation (2.3), and (c3)

$$
\begin{aligned}
\varepsilon\left\|x_{n}\right\|^{2} & \leq \varepsilon\left\langle J x_{n}, x_{n}\right\rangle+\left\langle T_{t_{n}} x_{n}, x_{n}\right\rangle=-s_{n} \Lambda\left\langle C x_{n}, x_{n}\right\rangle \\
& \leq s_{n} \Lambda \psi\left(\left\|x_{n}\right\|\right) \leq s_{n} \Lambda \psi(S)
\end{aligned}
$$

and so $x_{n} \rightarrow 0 \in \Omega$; but $x_{n} \in \partial \Omega$, which is a contradiction. Since we have the inequality

$$
\left\langle T_{t_{n}} x_{n}, x_{n}\right\rangle=-s_{n} \Lambda\left\langle C x_{n}, x_{n}\right\rangle-\varepsilon\left\langle J x_{n}, x_{n}\right\rangle \leq \Lambda \psi(S),
$$

Lemma 1.3 implies that the sequence $\left\{T_{t_{n}} x_{n}\right\}$ is bounded in the reflexive Banach space $X^{*}$. Passing to a subsequence, if necessary, we may suppose that $T_{t_{n}} x_{n} \rightarrow v^{*}$ for some $v^{*} \in X^{*}$. Set

$$
u^{*}:=\frac{1}{s_{0} \Lambda}\left(v^{*}+\varepsilon j^{*}\right) .
$$

By equation (2.3), we have $C x_{n} \rightarrow-u^{*}$ and hence

$$
\lim _{n \rightarrow \infty}\left\langle C x_{n}+u^{*}, y\right\rangle=0 \quad \text { for every } y \in L\left\{F_{n}\right\} .
$$

Recall that if two operators $A_{1}: D\left(A_{1}\right) \subset X \rightarrow 2^{X^{*}}$ and $A_{2}: D\left(A_{2}\right) \subset X \rightarrow 2^{X^{*}}$ are maximal monotone and $D\left(A_{1}\right) \cap \operatorname{int} D\left(A_{2}\right) \neq \emptyset$, then the sum $A_{1}+A_{2}: D\left(A_{1}\right) \cap D\left(A_{2}\right) \rightarrow 2^{X^{*}}$ is also maximal monotone; see [5, Theorem 32.I]. Since $T+\varepsilon J$ is thus maximal monotone and $T_{t_{n}} x_{n}+\varepsilon J x_{n} \rightarrow v^{*}+\varepsilon j^{*}$, Lemma 1.2(a) says that

$$
\liminf _{n \rightarrow \infty}\left\langle T_{t_{n}} x_{n}+\varepsilon J x_{n}, x_{n}-x_{0}\right\rangle \geq 0 .
$$

From equations (2.3), (2.5), and the equality

$$
\begin{aligned}
\left\langle C x_{n}+u^{*}, x_{n}\right\rangle= & \left\langle C x_{n}+\frac{1}{s_{n} \Lambda}\left(T_{t_{n}} x_{n}+\varepsilon J x_{n}\right), x_{n}\right\rangle-\left\langle\frac{1}{s_{n} \Lambda}\left(T_{t_{n}} x_{n}+\varepsilon J x_{n}\right), x_{n}-x_{0}\right\rangle \\
& -\left\langle\frac{1}{s_{n} \Lambda}\left(T_{t_{n}} x_{n}+\varepsilon J x_{n}\right), x_{0}\right\rangle+\left\langle u^{*}, x_{n}\right\rangle
\end{aligned}
$$


it follows that

$$
\begin{aligned}
\limsup _{n \rightarrow \infty}\left\langle C x_{n}+u^{*}, x_{n}\right\rangle & \leq-\liminf _{n \rightarrow \infty} \frac{1}{s_{n} \Lambda}\left\langle T_{t_{n}} x_{n}+\varepsilon J x_{n}, x_{n}-x_{0}\right\rangle \\
& \leq 0 .
\end{aligned}
$$

Since the operator $C$ satisfies the condition $\left(S_{+}\right)_{D(C)}$, we find from equations (2.4) and (2.6) that $x_{n} \rightarrow x_{0} \in D(C)$ and $C x_{0}+u^{*}=0$. Since $\lim _{n \rightarrow \infty}\left\langle T_{t_{n}} x_{n}, x_{n}-x_{0}\right\rangle=0$, Lemma 1.2(b) tells us that $x_{0} \in D(T)$ and $v^{*} \in T x_{0}$. From $J x_{n} \rightarrow J x_{0}=j^{*}$, we get

$$
v^{*}+s_{0} \Lambda C x_{0}+\varepsilon j^{*}=0 \quad \text { or } \quad 0 \in T x_{0}+s_{0} \Lambda C x_{0}+\varepsilon J x_{0},
$$

which contradicts our boundary condition equation (2.1). Consequently, we have proven our first assertion: that there exists a number $t_{0}>0$ such that

$$
H_{1}(t, s, x) \neq 0 \quad \text { for any }(t, s) \in\left(0, t_{0}\right] \times[0,1] \text { and all } x \in D(C) \cap \partial \Omega \text {. }
$$

In the next step, we want to show that for each fixed $t \in\left(0, t_{0}\right]$, the degree $d\left(H_{1}(t, s, \cdot)\right.$, $\Omega, 0)$ is independent of $s \in[0,1]$, where $d$ denotes the Kartsatos-Skrypnik degree from [12]. Fix $t \in\left(0, t_{0}\right]$. For $s \in[0,1]$, let $A_{s}: D\left(A_{s}\right) \subset X \rightarrow X^{*}$ be defined by

$$
A_{s} x:=H_{1}(t, s, x)=T_{t} x+s \Lambda C x+\varepsilon J x,
$$

where $D\left(A_{s}\right)=X$ for $s=0$ and $D\left(A_{s}\right)=D(C)$ for $s \in(0,1]$. First of all, for every finitedimensional space $F \subset L\left\{F_{j}\right\}$ and every $v \in L\left\{F_{j}\right\}$, the function $\tilde{a}(F, v): F \times[0,1] \rightarrow \mathbb{R}$, defined by $\tilde{a}(F, v)(x, s)=\left\langle A_{s} x, v\right\rangle$, is continuous on $F \times[0,1]$ because the operators $T_{t}$ and $J$ are continuous and $C$ satisfies the condition (c2). To show that the family $\left\{A_{s}\right\}$ satisfies the condition $\left(S_{+}\right)_{0, L}^{(s)}$, we assume that $\left\{s_{n}\right\}$ is a sequence in $[0,1]$ and $\left\{x_{n}\right\}$ is a sequence in $L\left\{F_{n}\right\}$ such that $s_{n} \rightarrow s_{0}, x_{n} \rightarrow x_{0}$, and

$$
\limsup _{n \rightarrow \infty}\left\langle A_{s_{n}} x_{n}, x_{n}\right\rangle \leq 0 \quad \text { and } \quad \lim _{n \rightarrow \infty}\left\langle A_{s_{n}} x_{n}, y\right\rangle=0
$$

for every $y \in L\left\{F_{n}\right\}$, where $s_{0} \in[0,1]$ and $x_{0} \in X$. By Lemma 1.1(a), the sequence $\left\{T_{t} x_{n}\right\}$ is bounded in $X^{*}$. So we may suppose without loss of generality that $T_{t} x_{n} \rightarrow v^{*}$ and $J x_{n} \rightarrow j^{*}$ for some $v^{*}, j^{*} \in X^{*}$. There are two cases to consider. If $s_{0}=0$, then we have

$$
\varepsilon\left\|x_{n}\right\|^{2} \leq \varepsilon\left\langle J x_{n}, x_{n}\right\rangle+\left\langle T_{t} x_{n}, x_{n}\right\rangle \leq\left\langle A_{s_{n}} x_{n}, x_{n}\right\rangle+s_{n} \Lambda \psi(S)
$$

which implies along with equation (2.7)

$$
\varepsilon \limsup _{n \rightarrow \infty}\left\|x_{n}\right\|^{2} \leq \lim _{n \rightarrow \infty} s_{n} \Lambda \psi(S)=0
$$

where $S$ is an upper bound for the sequence $\left\{\left\|x_{n}\right\|\right\}$. Hence it follows that $x_{n} \rightarrow 0, x_{0}=0 \in$ $X=D\left(A_{s_{0}}\right)$, and $A_{s_{0}} x_{0}=0$. Now let $s_{0} \in(0,1]$. We may suppose that $s_{n}>0$ for all $n \in \mathbb{N}$. Set 
$\tilde{s}_{n}:=1 /\left(s_{n} \Lambda\right)$ and $\tilde{s}:=1 /\left(s_{0} \Lambda\right)$. The relation (2.7) can be expressed in the form

$$
\begin{aligned}
& \limsup _{n \rightarrow \infty}\left\langle C x_{n}+\tilde{s}_{n}\left(T_{t}+\varepsilon J\right) x_{n}, x_{n}\right\rangle \leq 0, \\
& \lim _{n \rightarrow \infty}\left\langle C x_{n}+\tilde{s}_{n}\left(T_{t}+\varepsilon J\right) x_{n}, y\right\rangle=0 \quad \text { for every } y \in L\left\{F_{n}\right\} .
\end{aligned}
$$

From the second part of equation (2.8), it is obvious that

$$
\lim _{n \rightarrow \infty}\left\langle C x_{n}+\tilde{s}\left(v^{*}+\varepsilon j^{*}\right), y\right\rangle=0 \quad \text { for every } y \in L\left\{F_{n}\right\}
$$

By the monotonicity of the operator $T_{t}+\varepsilon J$, we have

$$
\liminf _{n \rightarrow \infty}\left\langle\left(T_{t}+\varepsilon J\right) x_{n}, x_{n}-x_{0}\right\rangle \geq \liminf _{n \rightarrow \infty}\left\langle\left(T_{t}+\varepsilon J\right) x_{0}, x_{n}-x_{0}\right\rangle=0
$$

Hence it follows from the first part of equation (2.8) and from equation (2.10) that

$$
\begin{aligned}
\limsup _{n \rightarrow \infty}\left\langle C x_{n}+\tilde{s}\left(v^{*}+\varepsilon j^{*}\right), x_{n}\right\rangle & \leq-\liminf _{n \rightarrow \infty} \tilde{s}_{n}\left\langle\left(T_{t}+\varepsilon J\right) x_{n}, x_{n}-x_{0}\right\rangle \\
& \leq 0
\end{aligned}
$$

Since the operator $C$ satisfies the condition $\left(S_{+}\right)_{L}$, we find from equations (2.9) and (2.11) that

$$
x_{n} \rightarrow x_{0}, \quad x_{0} \in D(C)=D\left(A_{s_{0}}\right) \quad \text { and } \quad C x_{0}+\tilde{s}\left(v^{*}+\varepsilon j^{*}\right)=0 .
$$

By the demicontinuity of the operators $T_{t}$ and $J$, we have

$$
T_{t} x_{n} \rightarrow T_{t} x_{0}=v^{*} \quad \text { and } J x_{n} \rightarrow J x_{0}=j^{*}
$$

and hence

$$
A_{s_{0}} x_{0}=T_{t} x_{0}+s_{0} \Lambda C x_{0}+\varepsilon J x_{0}=0 .
$$

Consequently, the family $\left\{A_{s}\right\}$ satisfies the condition $\left(S_{+}\right)_{0, L}^{(s)}$, as required.

Since $A_{s}(x) \neq 0$ for all $(s, x) \in[0,1] \times\left(D\left(A_{s}\right) \cap \partial \Omega\right)$, we see, in view of Theorem A of [18], that the degree $d\left(A_{s}, \Omega, 0\right)$ is independent of the choice of $s \in[0,1]$. Until now, we have shown that for each fixed $t \in\left(0, t_{0}\right]$, the degree $d\left(H_{1}(t, s, \cdot), \Omega, 0\right)$ is constant for all $s \in[0,1]$. Notice that $T+\varepsilon J$ is maximal monotone and strongly quasibounded, $0 \in(T+\varepsilon J)(0)$, and

$$
H(s, x)=(T+\varepsilon J) x+s \Lambda C x \not \ngtr 0 \quad \text { for all } s \in[0,1] \text { and all } x \in D(T+C) \cap \partial \Omega \text {. }
$$

Combining this with our first assertion above, Theorem 2 of [18] says that for each fixed $s \in[0,1]$, the degree $d\left(T_{t}+s \Lambda C+\varepsilon J, \Omega, 0\right)$ is constant for all $t \in\left(0, t_{0}\right]$. If deg denotes the degree introduced in [18], then for every $s \in[0,1]$, we have

$$
\operatorname{deg}(T+s \Lambda C+\varepsilon J, \Omega, 0)=\mathrm{d}\left(T_{t}+s \Lambda C+\varepsilon J, \Omega, 0\right) \quad \text { for } t \in\left(0, t_{0}\right]
$$


and hence

$$
\begin{aligned}
\operatorname{deg}(T+s \Lambda C+\varepsilon J, \Omega, 0) & =\mathrm{d}\left(A_{s}, \Omega, 0\right)=\mathrm{d}\left(A_{0}, \Omega, 0\right) \\
& =\mathrm{d}\left(T_{t}+\varepsilon J, \Omega, 0\right)=1,
\end{aligned}
$$

where the last equality follows from Theorem 3 in [23]. Thus, for all $s \in(0,1]$, the inclusion

$$
0 \in T x+s \Lambda C x+\varepsilon J x
$$

has a solution in $D(T+C) \cap \Omega$, which contradicts property $(\mathcal{P})$. We conclude that statement (a) is true.

(b) Let $\left\{\varepsilon_{n}\right\}$ be a sequence in $\left(0, \varepsilon_{0}\right]$ such that $\varepsilon_{n} \rightarrow 0$. According to statement (a), there exists a sequence $\left\{\left(\lambda_{\varepsilon_{n}}, x_{\varepsilon_{n}}\right)\right\}$ in $(0, \Lambda] \times(D(T+C) \cap \partial \Omega)$ such that

$$
u_{\varepsilon_{n}}^{*}+\lambda_{\varepsilon_{n}} C x_{\varepsilon_{n}}+\varepsilon_{n} J x_{\varepsilon_{n}}=0
$$

where $u_{\varepsilon_{n}}^{*} \in T x_{\varepsilon_{n}}$. If we set $\lambda_{n}:=\lambda_{\varepsilon_{n}}, x_{n}:=x_{\varepsilon_{n}}$, and $u_{n}^{*}:=u_{\varepsilon_{n}}^{*}$, it can be rewritten in the form

$$
u_{n}^{*}+\lambda_{n} C x_{n}+\varepsilon_{n} J x_{n}=0 .
$$

Notice that the sequence $\left\{u_{n}^{*}\right\}$ is bounded in $X^{*}$. This follows from the strong quasiboundedness of the operator $T$ together with the inequality

$$
\left\langle u_{n}^{*}, x_{n}\right\rangle=-\lambda_{n}\left\langle C x_{n}, x_{n}\right\rangle-\varepsilon_{n}\left\langle J x_{n}, x_{n}\right\rangle \leq \Lambda \psi(S)
$$

where $S$ is an upper bound for the sequence $\left\{\left\|x_{n}\right\|\right\}$. From equation (2.12), $\left\{\lambda_{n} C x_{n}\right\}$ is bounded in $X^{*}$. Without loss of generality, we may suppose that

$$
\lambda_{n} \rightarrow \lambda_{0}, \quad x_{n} \rightarrow x_{0}, \quad \text { and } \quad u_{n}^{*} \rightarrow u_{0}^{*}
$$

where $\lambda_{0} \in[0, \Lambda], x_{0} \in X$, and $u_{0}^{*} \in X^{*}$. Note that the limit $\lambda_{0}$ belongs to $(0, \Lambda]$. In fact, if $\lambda_{0}=0$, then the boundedness of the set $C(D(C) \cap \partial \Omega)$ implies that $\lambda_{n} C x_{n} \rightarrow 0$ and so by equation (2.12) $u_{n}^{*} \rightarrow 0$. Since the maximal monotone operator $T$ satisfies the condition $\left(S_{q}\right)$ on $D(T) \cap \partial \Omega$, we find from equation (2.13) and Lemma 1.2(b) that $x_{n} \rightarrow x_{0} \in \partial \Omega, x_{0} \in$ $D(T)$, and $0 \in T x_{0}$, which contradicts the hypothesis that $0 \notin T(D(T) \cap \partial \Omega)$. As $C x_{n} \rightarrow$ $\left(-1 / \lambda_{0}\right) u_{0}^{*}$, we have

$$
\lim _{n \rightarrow \infty}\left\langle C x_{n}+\frac{1}{\lambda_{0}} u_{0}^{*}, y\right\rangle=0 \quad \text { for every } y \in L\left\{F_{n}\right\} .
$$

From equation (2.12) it follows that

$$
\begin{aligned}
\limsup _{n \rightarrow \infty}\left\langle C x_{n}+\frac{1}{\lambda_{0}} u_{0}^{*}, x_{n}\right\rangle & \leq-\frac{1}{\lambda_{0}} \liminf _{n \rightarrow \infty}\left\langle u_{n}^{*}+\varepsilon_{n} J x_{n}, x_{n}-x_{0}\right\rangle \\
& \leq 0,
\end{aligned}
$$

where the last inequality follows from Lemma 1.2(a). Since the operator $C$ satisfies the condition $\left(S_{+}\right)_{D(C)}$, we obtain from equations (2.14) and (2.15) $x_{n} \rightarrow x_{0} \in D(C)$ and $\lambda_{0} C x_{0}+$ 
$u_{0}^{*}=0$. By the maximal monotonicity of the operator $T$, we have $x_{0} \in D(T)$ and $u_{0}^{*} \in T x_{0}$. We conclude that

$$
0 \in T x_{0}+\lambda_{0} C x_{0} \quad \text { and } \quad x_{0} \in D(T+C) \cap \partial \Omega
$$

This completes the proof.

Remark 2.2 (a) In Theorem 2.1, it is inevitable that the set $C(D(C) \cap \partial \Omega)$ is assumed to be bounded because it does not hold in general that if $\lambda_{n} \rightarrow 0$ then $\lambda_{n} C x_{n} \rightarrow 0$.

(b) When $C$ is quasibounded and satisfies the condition $\left(\tilde{S}_{+}\right)$, it was studied in [9, Theorem 4] by using Kartsatos-Skrypnik degree theory for $\left(\tilde{S}_{+}\right)$-perturbations of maximal monotone operators developed in [13]. For the case where $C$ is generalized pseudomonotone in place of the condition $\left(\tilde{S}_{+}\right)$, we refer to [20, Theorem 2.1].

From Theorem 2.1, we get the following eigenvalue result in the case when the operator $C$ satisfies the condition $\left(S_{+}\right)$.

Corollary 2.3 Let $T, \Omega, L, \Lambda, \varepsilon_{0}$ be as in Theorem 2.1. Suppose that $C: X \rightarrow X^{*}$ is a strongly quasibounded demicontinuous operator such that

(c1') C satisfies the condition $\left(S_{+}\right)$on $X$,

(c2) for every $F \in \mathcal{F}(L)$ and $v \in L$, the function $c(F, v): F \rightarrow \mathbb{R}$, defined by $c(F, v)(x)=$ $\langle C x, v\rangle$, is continuous on $F$, and

(c3) there exists a nondecreasing function $\psi:[0, \infty) \rightarrow[0, \infty)$ such that

$$
\langle C x, x\rangle \geq-\psi(\|x\|) \text { for all } x \in X .
$$

Then the following statements hold:

(a) If property $(\mathcal{P})$ is fulfilled for a given $\varepsilon>0$, then there exists a $\left(\lambda_{\varepsilon}, x_{\varepsilon}\right) \in(0, \Lambda] \times(D(T) \cap \partial \Omega)$ such that $0 \in T x_{\varepsilon}+\lambda_{\varepsilon} C x_{\varepsilon}+\varepsilon J x_{\varepsilon}$.

(b) If property $(\mathcal{P})$ is fulfilled for every $\varepsilon \in\left(0, \varepsilon_{0}\right]$, T satisfies the condition $\left(S_{q}\right)$ on $D(T) \cap \partial \Omega$ and $0 \notin T(D(T) \cap \partial \Omega)$, then the inclusion $0 \in T x+\lambda C x$ has a solution in $(0, \Lambda] \times(D(T) \cap \partial \Omega)$.

Proof Statement (a) follows immediately from Theorem 2.1 if we only show that the operator $C$ satisfies the condition $\left(S_{+}\right)_{D(C)}$ with $D(C)=X$. To do this, let $h \in X^{*}$ be given and suppose that $\left\{x_{n}\right\}$ is any sequence in $X$ such that

$$
x_{n}-x_{0}, \quad \limsup _{n \rightarrow \infty}\left\langle C x_{n}-h, x_{n}\right\rangle \leq 0, \quad \text { and } \quad \lim _{n \rightarrow \infty}\left\langle C x_{n}-h, y\right\rangle=0
$$

for every $y \in L\left\{F_{n}\right\}$. Then $\left\{\left\langle C x_{n}, x_{n}\right\rangle\right\}$ is obviously bounded from above. By the strong quasiboundedness of the operator $C$, the sequence $\left\{C x_{n}\right\}$ is bounded in $X^{*}$. Since $L\left\{F_{n}\right\}$ is dense in the reflexive Banach space $X$, it follows from the third one of equation (2.16) that $C x_{n} \rightarrow h$. Hence we obtain from the first and second one of equation (2.16)

$$
\begin{aligned}
& \limsup _{n \rightarrow \infty}\left\langle C x_{n}, x_{n}-x_{0}\right\rangle \\
& \quad \leq \limsup _{n \rightarrow \infty}\left\langle C x_{n}-h, x_{n}\right\rangle-\lim _{n \rightarrow \infty}\left\langle C x_{n}-h, x_{0}\right\rangle+\lim _{n \rightarrow \infty}\left\langle h, x_{n}-x_{0}\right\rangle \leq 0 .
\end{aligned}
$$


Since $C$ satisfies the condition $\left(S_{+}\right)$on $X$ and is demicontinuous, we have

$$
x_{n} \rightarrow x_{0} \in X \text { and } C x_{0}-h=0 .
$$

Thus, the operator $C$ satisfies the condition $\left(S_{+}\right)_{D(C)}$ with $D(C)=X$.

(b) Let $\left\{\varepsilon_{n}\right\}$ be a sequence in $\left(0, \varepsilon_{0}\right]$ such that $\varepsilon_{n} \rightarrow 0$. In view of (a), there exists a sequence $\left\{\left(\lambda_{n}, x_{n}\right)\right\}$ in $(0, \Lambda] \times(D(T) \cap \partial \Omega)$ such that

$$
u_{n}^{*}+\lambda_{n} C x_{n}+\varepsilon_{n} J x_{n}=0
$$

where $u_{n}^{*} \in T x_{n}$. Notice that the sequence $\left\{C x_{n}\right\}$ is bounded in $X^{*}$ and so is $\left\{u_{n}^{*}\right\}$. This follows from the strong quasiboundedness of the operator $C$ and the inequality

$$
\left\langle C x_{n}, x_{n}\right\rangle=-\frac{1}{\lambda_{n}}\left\langle u_{n}^{*}, x_{n}\right\rangle-\frac{\varepsilon_{n}}{\lambda_{n}}\left\langle J x_{n}, x_{n}\right\rangle \leq 0 .
$$

We may suppose that $\lambda_{n} \rightarrow \lambda_{0}, x_{n} \rightarrow x_{0}$, and $u_{n}^{*} \rightarrow u_{0}^{*}$, where $\lambda_{0} \in[0, \Lambda], x_{0} \in X$, and $u_{0}^{*} \in X^{*}$. Note that $\lambda_{0}$ belongs to $(0, \Lambda]$. Indeed, if $\lambda_{0}=0$, then we have by the boundedness of $\left\{C x_{n}\right\}$ and equation (2.17) $u_{n}^{*} \rightarrow 0$ and hence by the condition $\left(S_{q}\right) x_{n} \rightarrow x_{0} \in D(T)$ and $0 \in T x_{0}$, which contradicts the hypothesis $0 \notin T(D(T) \cap \partial \Omega)$. The rest of the proof proceeds analogously as in the proof of Theorem 2.1.

Remark 2.4 (a) The boundedness assumption on the set $C(D(C) \cap \partial \Omega)$ is unnecessary in Corollary 2.3, provided that the operator $C$ is strongly quasibounded.

(b) An analogous result to Corollary 2.3 can be found in [9, Corollary 1], where the operator $C$ is supposed to be bounded.

We close this section by exhibiting a simple example of operators $A$ satisfying the condition $\left(S_{+}\right)_{D(A)}$.

Let $G$ be a bounded open set in $\mathbb{R}^{N}$. Let $1<p<\infty$ and $X=W_{0}^{1, p}(G)$. Define the two operators $A_{1}, A_{2}: X \rightarrow X^{*}$ by

$$
\begin{aligned}
& \left\langle A_{1} u, v\right\rangle=\sum_{i=1}^{N} \int_{G}\left|\frac{\partial u}{\partial x_{i}}\right|^{p-2} \frac{\partial u}{\partial x_{i}} \frac{\partial v}{\partial x_{i}} d x, \\
& \left\langle A_{2} u, v\right\rangle=\int_{G}|u|^{p-2} u v d x .
\end{aligned}
$$

Then the operator $A_{1}$ is clearly bounded and continuous, and it satisfies the condition $\left(S_{+}\right)$ on $X$. The operator $A_{2}$ is compact; see [24, Theorem 2.2] and [5, Proposition 26.10]. In particular, the sum $A:=A_{1}+A_{2}$ satisfies the condition $\left(S_{+}\right)_{D(A)}$ with $D(A)=X$.

\section{Fredholm alternative}

In this section, we present a variant of the Fredholm alternative for strongly quasibounded maximal monotone operators, by applying Kartsatos-Quarcoo degree theory as in Section 2.

Given $\gamma>0$, an operator $A: D(A) \subset X \rightarrow X^{*}$ is said to be positively homogeneous of degree $\gamma$ on a set $M \subset D(A)$ if $A(r x)=r^{\gamma} A x$ for all $x \in M$ and all $r>0$. For example, the 
duality operator $J_{\varphi}: X \rightarrow X^{*}$ is positively homogeneous of degree $\gamma$ on $X$ if $\varphi(t)=t^{\gamma}$ for $t \in[0, \infty)$. In addition, the operators $A_{1}$ and $A_{2}$ given at the end of Section 2 are positively homogeneous of degree $p-1$ on $X=W_{0}^{1, p}(G)$.

Theorem 3.1 Let $L$ be a dense subspace of $X$ and let $\lambda, \gamma \in[1, \infty)$ be given. Suppose that $T: D(T)=L \rightarrow X^{*}$ is an operator and $C: D(C) \subset X \rightarrow X^{*}$ is an operator with $L \subset D(C)$ and $C(0)=0$ such that

(t1) $T$ is maximal monotone and strongly quasibounded with $T(0)=0$,

(t2) $\lambda T x+C x+\mu J_{\varphi} x=0$ implies $x=0$ for every $\mu \geq 0$, where $\varphi(t)=t^{\gamma}$,

(c1) $C$ satisfies the condition $\left(S_{+}\right)_{L}$,

(c2) for every $F \in \mathcal{F}(L)$ and $v \in L$, the function $c(F, v): F \rightarrow \mathbb{R}$, defined by $c(F, v)(x)=\langle C x, v\rangle$, is continuous on $F$, and

(c3) there exists a nondecreasing function $\psi:[0, \infty) \rightarrow[0, \infty)$ such that

$$
\langle C x, x\rangle \geq-\psi(\|x\|) \text { for all } x \in D(C)
$$

If the operators $T$ and $C$ are positively homogeneous of degree $\gamma$ on $L$, then the operator $\lambda T+C$ is surjective.

Proof Let $p^{*}$ be an arbitrary but fixed element of $X^{*}$. For each fixed $\varepsilon>0$, consider a family of operators $A_{t}: D\left(A_{t}\right) \subset X \rightarrow X^{*}, t \in[0,1]$ given by

$$
A_{t}(x):=H(t, x):=t\left(\lambda T x+C x+\varepsilon J_{\varphi} x-p^{*}\right)+(1-t) \varepsilon J_{\varphi} x,
$$

where $D\left(A_{t}\right)=X$ for $t=0$ and $D\left(A_{t}\right)=L$ for $t \in(0,1]$. The first aim is to prove that the set of all solutions of the equation $H(t, x)=0$ is bounded, independent of $t \in[0,1]$. If $t=0$, then $H(0, x)=\varepsilon J_{\varphi} x=0$ implies $x=0$. It suffices to show that $\{(t, x) \in(0,1] \times L: H(t, x)=0\}$ is bounded. Assume the contrary; then there exist sequences $\left\{t_{n}\right\}$ in $(0,1]$ and $\left\{x_{n}\right\}$ in $L$ such that $t_{n} \rightarrow t_{0} \in[0,1],\left\|x_{n}\right\| \rightarrow \infty$, and

$$
t_{n}\left(\lambda T x_{n}+C x_{n}+\varepsilon J_{\varphi} x_{n}-p^{*}\right)+\left(1-t_{n}\right) \varepsilon J_{\varphi} x_{n}=0,
$$

which can be written as

$$
\lambda T x_{n}+C x_{n}-p^{*}+\frac{\varepsilon}{t_{n}} J_{\varphi} x_{n}=0 .
$$

We may suppose that $\left\|x_{n}\right\| \geq 1$ for all $n \in \mathbb{N}$. Since the operators $T, C$, and $J_{\varphi}$ are positively homogeneous of degree $\gamma$, it follows from equation (3.1) that

$$
\lambda T\left(\frac{x_{n}}{\left\|x_{n}\right\|}\right)+C\left(\frac{x_{n}}{\left\|x_{n}\right\|}\right)-\frac{1}{\left\|x_{n}\right\|^{\gamma}} p^{*}+\frac{\varepsilon}{t_{n}} J_{\varphi}\left(\frac{x_{n}}{\left\|x_{n}\right\|}\right)=0 .
$$

Setting $u_{n}:=x_{n} /\left\|x_{n}\right\|$ and $q_{n}:=1 / t_{n}$, we have $\left\|u_{n}\right\|=1, q_{n}>0$, and

$$
\lambda T u_{n}+C u_{n}-\frac{1}{\left\|x_{n}\right\|^{\gamma}} p^{*}+q_{n} \varepsilon J_{\varphi} u_{n}=0
$$


Then we obtain from equation (3.2) and (c3)

$$
\begin{aligned}
\lambda\left\langle T u_{n}, u_{n}\right\rangle & =-\left\langle C u_{n}, u_{n}\right\rangle+\frac{1}{\left\|x_{n}\right\|^{\gamma}}\left\langle p^{*}, u_{n}\right\rangle-q_{n} \varepsilon\left\langle J_{\varphi} u_{n}, u_{n}\right\rangle \\
& \leq \psi(1)+\left\|p^{*}\right\| .
\end{aligned}
$$

Hence the strong quasiboundedness of $T$ implies that the sequence $\left\{T u_{n}\right\}$ is bounded in $X^{*}$. There are two cases to consider. If $t_{0}=0$, then $q_{n} \rightarrow \infty,\left\langle J_{\varphi} u_{n}, u_{n}\right\rangle=1$, and the monotonicity of $T$ with $T(0)=0$ implies

$$
0 \leq \lambda\left\langle T u_{n}, u_{n}\right\rangle \leq \psi(1)+\left\|p^{*}\right\|-q_{n} \varepsilon \rightarrow-\infty,
$$

which is a contradiction. Now let $t_{0}>0$ and set $q_{0}:=1 / t_{0}$. Without loss of generality, we may suppose that

$$
u_{n} \rightarrow u_{0}, \quad T u_{n} \rightarrow v^{*}, \quad \text { and } J_{\varphi} u_{n} \rightarrow j^{*},
$$

where $u_{0} \in X, v^{*} \in X^{*}$, and $j^{*} \in X^{*}$. By equation (3.2), we have $C u_{n} \rightarrow-\lambda v^{*}-q_{0} \varepsilon j^{*}$ and hence

$$
\lim _{n \rightarrow \infty}\left\langle C u_{n}+\lambda v^{*}+q_{0} \varepsilon j^{*}, y\right\rangle=0 \quad \text { for every } y \in L\left\{F_{n}\right\} .
$$

Since the operator $\lambda T+q_{0} \varepsilon J_{\varphi}$ is maximal monotone, we have

$$
\liminf _{n \rightarrow \infty}\left\langle\lambda T u_{n}+q_{n} \varepsilon J_{\varphi} u_{n}, u_{n}-u_{0}\right\rangle \geq 0
$$

In fact, if equation (3.4) is false, then there is a subsequence of $\left\{u_{n}\right\}$, denoted again by $\left\{u_{n}\right\}$, such that

$$
\lim _{n \rightarrow \infty}\left\langle\lambda T u_{n}+q_{n} \varepsilon J_{\varphi} u_{n}, u_{n}-u_{0}\right\rangle<0 .
$$

Hence it is clear that

$$
\limsup _{n \rightarrow \infty}\left\langle\lambda T u_{n}+q_{n} \varepsilon J_{\varphi} u_{n}, u_{n}\right\rangle<\left\langle\lambda v^{*}+q_{0} \varepsilon j^{*}, u_{0}\right\rangle
$$

For every $u \in D(T)$, we have, by the monotonicity of the operator $\lambda T+q_{n} \varepsilon J_{\varphi}$,

$$
\begin{aligned}
& \liminf _{n \rightarrow \infty}\left\langle\lambda T u_{n}+q_{n} \varepsilon J_{\varphi} u_{n}, u_{n}\right\rangle \\
& \quad \geq \liminf _{n \rightarrow \infty}\left[\left\langle\lambda T u_{n}+q_{n} \varepsilon J_{\varphi} u_{n}, u\right\rangle+\left\langle\lambda T u+q_{n} \varepsilon J_{\varphi} u, u_{n}-u\right\rangle\right] \\
& \quad=\left\langle\lambda v^{*}+q_{0} \varepsilon j^{*}, u\right\rangle+\left\langle\lambda T u+q_{0} \varepsilon J_{\varphi} u, u_{0}-u\right\rangle,
\end{aligned}
$$

which implies along with equation (3.5)

$$
\left\langle\lambda \nu^{*}+q_{0} \varepsilon j^{*}-\left(\lambda T u+q_{0} \varepsilon J_{\varphi} u\right), u_{0}-u\right\rangle>0 .
$$


By the maximal monotonicity of $\lambda T+q_{0} \varepsilon J_{\varphi}$, we have $u_{0} \in D(T)$ and $\left(\lambda T+q_{0} \varepsilon J_{\varphi}\right) u_{0}=$ $\lambda v^{*}+q_{0} \varepsilon j^{*}$. Letting $u=u_{0} \in D(T)$ in equation (3.6), we get a contradiction. Thus, equation (3.4) is true.

Furthermore, equation (3.4) implies, because of $\left(1 /\left\|x_{n}\right\|^{\gamma}\right) p^{*} \rightarrow 0$, that

$$
\liminf _{n \rightarrow \infty}\left\langle\lambda T u_{n}-\frac{1}{\left\|x_{n}\right\|^{\gamma}} p^{*}+q_{n} \varepsilon J_{\varphi} u_{n}, u_{n}-u_{0}\right\rangle \geq 0
$$

From equations (3.2), (3.7), and the equality

$$
\begin{aligned}
\left\langle C u_{n}+\lambda v^{*}+q_{0} \varepsilon j^{*}, u_{n}\right\rangle & \\
= & \left\langle C u_{n}+\lambda T u_{n}-\frac{1}{\left\|x_{n}\right\|^{\gamma}} p^{*}+q_{n} \varepsilon J_{\varphi} u_{n}, u_{n}\right\rangle \\
& -\left\langle\lambda T u_{n}-\frac{1}{\left\|x_{n}\right\|^{\gamma}} p^{*}+q_{n} \varepsilon J_{\varphi} u_{n}, u_{n}-u_{0}\right\rangle \\
& -\left\langle\lambda T u_{n}-\frac{1}{\left\|x_{n}\right\|^{\gamma}} p^{*}+q_{n} \varepsilon J_{\varphi} u_{n}, u_{0}\right\rangle+\left\langle\lambda v^{*}+q_{0} \varepsilon j^{*}, u_{n}\right\rangle
\end{aligned}
$$

it follows that

$$
\begin{aligned}
& \limsup _{n \rightarrow \infty}\left\langle C u_{n}+\lambda v^{*}+q_{0} \varepsilon j^{*}, u_{n}\right\rangle \\
& \quad \leq-\liminf _{n \rightarrow \infty}\left\langle\lambda T u_{n}-\frac{1}{\left\|x_{n}\right\| \gamma} p^{*}+q_{n} \varepsilon J_{\varphi} u_{n}, u_{n}-u_{0}\right\rangle \\
& \quad \leq 0 .
\end{aligned}
$$

Since the operator $C$ satisfies the condition $\left(S_{+}\right)_{L}$, we obtain from equations (3.3) and (3.8)

$$
u_{n} \rightarrow u_{0}, \quad u_{0} \in D(C), \quad \text { and } \quad C u_{0}+\lambda v^{*}+q_{0} \varepsilon j^{*}=0 .
$$

Since $T$ is maximal monotone and $J_{\varphi}$ is continuous, Lemma 1.2(b) implies that

$$
u_{0} \in D(T), \quad T u_{0}=v^{*}, \quad \text { and } \quad J_{\varphi} u_{0}=j^{*} .
$$

Therefore, we obtain

$$
\lambda T u_{0}+C u_{0}+q_{0} \varepsilon J_{\varphi} u_{0}=0 \text { and }\left\|u_{0}\right\|=1,
$$

which contradicts hypothesis (t2) with $\mu=q_{0} \varepsilon$. Thus, we have shown that $\{(t, x) \in[0,1] \times$ $L: H(t, x)=0\}$ is bounded.

So we can choose an open ball $B_{r}(0)$ in $X$ of radius $r>0$ centered at the origin 0 so that

$$
\{x \in L: H(t, x)=0 \text { for some } t \in[0,1]\} \subset B_{r}(0) \text {. }
$$

This means that $H(t, x)=A_{t}(x) \neq 0$ for all $(t, x) \in[0,1] \times\left(D\left(A_{t}\right) \cap \partial B_{r}(0)\right)$. Note that the operator $\tilde{T}_{\varepsilon}:=\lambda T+\varepsilon J_{\varphi}$ is maximal monotone, strongly quasibounded, $\tilde{T}_{\varepsilon}(0)=0$, and the operator $\tilde{C}:=C-p^{*}$ satisfies the condition $\left(S_{+}\right)_{L}$ and other conditions with $\tilde{c}(F, v)(x):=\langle\tilde{C} x, v\rangle$ 
for $x \in F$ and $\langle\tilde{C} x, x\rangle \geq-\tilde{\psi}(\|x\|)$ for $x \in D(\tilde{C})$, where $\tilde{\psi}(t):=\left(1+\left\|p^{*}\right\|\right) \max \{\psi(t), t\}$. Moreover, we know from Section 1 that the operator $\varepsilon J_{\varphi}$ is continuous, bounded and strictly monotone, and that it satisfies the condition $\left(S_{+}\right)$, and $\left\langle\varepsilon J_{\varphi} x, x\right\rangle=\varepsilon\|x\|^{\gamma+1}$ for $x \in X$.

Using the homotopy invariance property of the degree stated in [18, Theorem 3$]$, we have

$$
\operatorname{deg}\left(\lambda T+C+\varepsilon J_{\varphi}-p^{*}, B_{r}(0), 0\right)=\operatorname{deg}\left(\varepsilon J_{\varphi}, B_{r}(0), 0\right)=1
$$

Applying equation (3.9) with $\varepsilon=1 / n$, there exists a sequence $\left\{x_{n}\right\}$ in $L$ such that

$$
\lambda T x_{n}+C x_{n}+\frac{1}{n} J_{\varphi} x_{n}=p^{*} .
$$

Next, we show that the sequence $\left\{x_{n}\right\}$ is bounded in $X$. Indeed, assume on the contrary that there is a subsequence of $\left\{x_{n}\right\}$, denoted by $\left\{x_{n}\right\}$, such that $\left\|x_{n}\right\| \rightarrow \infty$. Dividing both sides of equation (3.10) by $\left\|x_{n}\right\|^{\gamma}$ and setting $u_{n}:=x_{n} /\left\|x_{n}\right\|$ and $w_{n}^{*}:=\lambda T u_{n}+C u_{n}$, we get

$$
\lambda T u_{n}+C u_{n}+\frac{1}{n} J_{\varphi} u_{n}=\frac{1}{\left\|x_{n}\right\|^{\gamma}} p^{*}
$$

and so $w_{n}^{*} \rightarrow 0$. Since $\lambda\left\langle T u_{n}, u_{n}\right\rangle=-\left\langle C u_{n}, u_{n}\right\rangle+\left\langle w_{n}^{*}, u_{n}\right\rangle \leq \psi(1)+\left\|w_{n}^{*}\right\|$ for all $n \in \mathbb{N}$, it follows from (t1) that the sequence $\left\{T u_{n}\right\}$ is bounded in $X^{*}$. We may suppose that $u_{n} \rightarrow u_{0}$ and $T u_{n} \rightarrow v^{*}$ for some $u_{0} \in X$ and some $v^{*} \in X^{*}$. As in the proof of equations (3.3) and (3.8) above, we can show that

$$
\limsup _{n \rightarrow \infty}\left\langle C u_{n}+\lambda v^{*}, u_{n}\right\rangle \leq 0 \text { and } \lim _{n \rightarrow \infty}\left\langle C u_{n}+\lambda v^{*}, y\right\rangle=0
$$

for every $y \in L\left\{F_{n}\right\}$. Since the operator $C$ satisfies the condition $\left(S_{+}\right)_{L}$, we obtain

$$
u_{n} \rightarrow u_{0}, \quad u_{0} \in D(C), \text { and } C u_{0}+\lambda v^{*}=0 .
$$

By Lemma 1.2(b), we have $u_{0} \in D(T)$ and $T u_{0}=v^{*}$ and hence

$$
\lambda T u_{0}+C u_{0}=0 \text { and }\left\|u_{0}\right\|=1,
$$

which contradicts hypothesis (t2) with $\mu=0$. Therefore, the sequence $\left\{x_{n}\right\}$ is bounded in $X$.

Combining this with equation (3.10), we know from (c3) and (t1) that the sequence $\left\{T x_{n}\right\}$ is also bounded in $X^{*}$. Thus we may suppose that $x_{n} \rightarrow x_{0}$ and $T x_{n} \rightarrow v_{0}^{*}$ for some $x_{0} \in X$ and some $v_{0}^{*} \in X^{*}$. From $C x_{n} \rightarrow-\lambda v_{0}^{*}+p^{*}$ and the maximal monotonicity of the operator $T$, we get as before

$$
\limsup _{n \rightarrow \infty}\left\langle C x_{n}+\lambda v_{0}^{*}-p^{*}, x_{n}\right\rangle \leq 0 \text { and } \lim _{n \rightarrow \infty}\left\langle C x_{n}+\lambda v_{0}^{*}-p^{*}, y\right\rangle=0
$$

for every $y \in L\left\{F_{n}\right\}$. Since the operator $C$ satisfies the condition $\left(S_{+}\right)_{L}$ and $T$ is maximal monotone, we conclude that

$$
x_{0} \in D(\lambda T+C) \text { and } \lambda T x_{0}+C x_{0}=p^{*} .
$$


As $p^{*} \in X^{*}$ was arbitrary, this says that the operator $\lambda T+C$ is surjective. This completes the proof.

Remark 3.2 An analogous result to Theorem 3.1 was investigated in [20, Theorem 4.1], where the method was to use Kartsatos-Skrypnik degree theory for quasibounded densely defined $\left(\tilde{S}_{+}\right)$-perturbations of maximal monotone operators, developed in [13]; see also [9, Theorem 5].

As a particular case of Theorem 3.1, we have another surjectivity result.

Corollary 3.3 Let L, T, and C be the same as in Theorem 3.1, except that hypothesis (t2) is replaced by

$\left(\mathrm{t} 2^{\prime}\right)\langle\lambda T x+C x, x\rangle \geq 0$ for all $x \in L$.

If $\lambda$ is not an eigenvalue for the pair $(T, C)$, that is, $\lambda T x+C x=0$ implies $x=0$, then the operator $\lambda T+C$ is surjective.

Proof Noting that

$$
\left\langle\lambda T x+C x+\mu J_{\varphi} x, x\right\rangle \geq \mu\left\langle J_{\varphi} x, x\right\rangle=\mu\|x\|^{\gamma+1} \geq 0
$$

for every $x \in L$ and $\mu>0$, it is clear that hypothesis (t2) in Theorem 3.1 is satisfied. Apply Theorem 3.1.

Competing interests

The authors declare that they have no competing interests.

Authors' contributions

$\mathrm{KI}$ conceived of the study and drafted the manuscript. BI participated in coordination. All authors approved the final manuscript.

\section{Acknowledgements}

This research was supported by the Basic Science Research Program through the National Research Foundation of Korea (NRF) funded by the Ministry of Education, Science and Technology (NRF-2012-0008345).

Received: 11 October 2013 Accepted: 16 December 2013 Published: 14 Jan 2014

\section{References}

1. Krasnosel'skii, MA: Topological Methods in the Theory of Nonlinear Integral Equations. Pergamon, New York (1964)

2. Zeidler, E: Nonlinear Functional Analysis and Its Applications III: Variational Methods and Optimization. Springer, New York (1985)

3. Minty, G: Monotone operators in Hilbert spaces. Duke Math. J. 29, 341-346 (1962)

4. Browder, FE: Nonlinear operators and nonlinear equations of evolution in Banach spaces. Nonlinear Functional Analysis (Proc. Sympos. Pure Math., Vol. XVIII, Part 2, Chicago, III., 1968), pp. 1-308. Am. Math. Soc., Providence (1976)

5. Zeidler, E: Nonlinear Functional Analysis and Its Applications II/B: Nonlinear Monotone Operators. Springer, New York (1990)

6. Guan, Z, Kartsatos, AG: On the eigenvalue problem for perturbations of nonlinear accretive and monotone operators in Banach spaces. Nonlinear Anal. 27, 125-141 (1996)

7. Kartsatos, AG: New results in the perturbation theory of maximal monotone and $m$-accretive operators in Banach spaces. Trans. Am. Math. Soc. 348, 1663-1707 (1996)

8. Kartsatos, AG, Skrypnik, IV: Normalized eigenvectors for nonlinear abstract and elliptic operators. J. Differ. Equ. 155, 443-475 (1999)

9. Kartsatos, AG, Skrypnik, IV: On the eigenvalue problem for perturbed nonlinear maximal monotone operators in reflexive Banach spaces. Trans. Am. Math. Soc. 358, 3851-3881 (2006)

10. Li, H-X, Huang, F-L: On the nonlinear eigenvalue problem for perturbations of monotone and accretive operators in Banach spaces. Sichuan Daxue Xuebao (J. Sichuan Univ.) 37, 303-309 (2000)

11. Browder, FE: Fixed point theory and nonlinear problems. Bull. Am. Math. Soc. 9, 1-39 (1983) 
12. Kartsatos, AG, Skrypnik, IV: Topological degree theories for densely defined mappings involving operators of type $\left(S_{+}\right)$. Adv. Differ. Equ. 4, 413-456 (1999)

13. Kartsatos, AG, Skrypnik, IV: A new topological degree theory for densely defined quasibounded $\left(\tilde{S}_{+}\right)$-perturbations of multivalued maximal monotone operators in reflexive Banach spaces. Abstr. Appl. Anal. 2005, 121-158 (2005)

14. Skrypnik, IV: Nonlinear Higher Order Elliptic Equations. Naukova Dumka, Kiev (1973) (Russian)

15. Skrypnik, IV: Methods for Analysis of Nonlinear Elliptic Boundary Value Problems. Transl., Ser. II., vol. 139. Am. Math. Soc., Providence (1994)

16. Browder, FE, Hess, P: Nonlinear mappings of monotone type in Banach spaces. J. Funct. Anal. 11, 251-294 (1972)

17. Berkovits, J: On the degree theory for densely defined mappings of class $\left(S_{+}\right)_{L}$. Abstr. Appl. Anal. 4, 141-152 (1999)

18. Kartsatos, AG, Quarcoo, J: A new topological degree theory for densely defined $\left(S_{+}\right)_{L}$-perturbations of multivalued maximal monotone operators in reflexive separable Banach spaces. Nonlinear Anal. 69, 2339-2354 (2008)

19. Brézis, H, Crandall, MG, Pazy, A: Perturbations of nonlinear maximal monotone sets in Banach space. Commun. Pure Appl. Math. 23, 123-144 (1970)

20. Kim, I-S, Bae, J-H: Eigenvalue results for pseudomonotone perturbations of maximal monotone operators. Cent. Eur. J. Math. 11, 851-864 (2013)

21. Petryshyn, WV: Approximation-Solvability of Nonlinear Functional and Differential Equations. Dekker, New York (1993)

22. Adhikari, DR, Kartsatos, AG: Topological degree theories and nonlinear operator equations in Banach spaces. Nonlinear Anal. 69, 1235-1255 (2008)

23. Browder, FE: Degree of mapping for nonlinear mappings of monotone type. Proc. Natl. Acad. Sci. USA 80, 1771-1773 (1983)

24. Schmitt, K, Sim, I: Bifurcation problems associated with generalized Laplacians. Adv. Differ. Equ. 9, 797-828 (2004)

10.1186/1029-242X-2014-21

Cite this article as: Kim and Bae: Eigenvalues of quasibounded maximal monotone operators. Journal of Inequalities and Applications 2014, 2014:21

\section{Submit your manuscript to a SpringerOpen ${ }^{\ominus}$ journal and benefit from:}

- Convenient online submission

- Rigorous peer review

- Immediate publication on acceptance

- Open access: articles freely available online

- High visibility within the field

- Retaining the copyright to your article 